\title{
Excitation of Optical Radiation by High Power Density Radio Beams
}

\author{
Lawrence R. Megill
}

\section{Contribution From the Central Radio Propagation Laboratory, National Bureau of Standards, Boulder, Colo.}

(Received May 14, 1964; revised June 15, 1964)

\begin{abstract}
We calculate the power density required to excite various states of $\mathrm{O}_{2}$ and $\mathrm{O}$ which may be expected to radiate light observable from the ground. These calculations are made by first calculating the electron energy distribution functions in the presence of the RF electric fields, and from this the rate of excitation of the radiating states. We discuss the feasibility of experiments to generate sufficient intensity to cause appreciable modification in the night airglow intensity. The possibility of performing experiments using the two body dissociative attachment of electrons is also considered, especially in regard to the performance of basic experiments in atomic physics.
\end{abstract}

\section{Introduction}

In the discussion of nonlinear processes in the ionosphere, the excitation of optical radiation by a radio beam is an extreme case. Yet, if transmitters sufficiently powerful to excite optical radiation in the ionosphere existed, a great deal of quantitative information concerning the constitution of the ionosphere could be collected on a routine basis. It is, therefore, of some importance to investigate the conditions under which detectable optical radiation might be excited.

This problem has been considered by others, Bailey [1959], Clavier [1961]; but not in the detail which we will apply. Authors in the past have estimated the intensity to be expected in the atmosphere based on laboratory data obtained in a discharge. Such a procedure is liable to considerable error, since conditions in a discharge differ greatly from those extant in the atmosphere. In particular, in discharges there exist many metastable states which change the power required to heat electrons and recombination processes which yield a great deal of the luminosity. In addition, the electron density in discharges is often such that the electron energy distribution is dominated by electron interactions, a condition which does not apply in the lower ionosphere, [Megill and Carleton, 1964]. We shall, in this paper, concern ourselves principally with the direct "heating" of the electrons already in existence in the ionosphere by means of electric fields and with the excitation of electronic states in the neutral gas by collisions with these electrons. The problem of "breaking down" the atmosphere will be discussed only briefly.

\section{Excitation of Optical Radiation}

If we know the distribution $f(u)$ of electrons witb energy, $u$, under the influence of electric and magnetic fields then we can calculate the total rate of excitation $\nu_{i j}$ of a particular state from

$$
\nu_{i j}=N_{i} N_{e} \int_{0}^{\infty} \sigma_{i j} v f(u) u^{\frac{1}{2}} d u
$$

where $N_{i}$ is the number density of the gas, $N_{e}$ is the number density of electrons, $\sigma_{i j}$ is the cross section for excitation to state $j, v$ is the electron velocity, and we have normalized the distribution function so that

$$
\int_{0}^{\infty} f(u) u^{\frac{1}{2}} d u=1
$$

Carleton and Megill [1962] have described the calculation of the electron energy distribution function for altitudes of interest here. It is shown that the distribution function may be determined by solving the following differential equation.

$$
[k T \delta \nu(u)+\gamma \alpha \nu(u)] \frac{\partial f}{\partial u}=-\delta \nu(u) f(u)-Q(u)
$$

where

$k$ is the Boltzmann constant.

$T$ is the temperature of the neutral particles.

$\nu$ is the elastic collision frequency $\Sigma N_{i} \sigma_{i} v$ where $N_{i}$ is the density of gas $i$ and $\sigma$ is the elastic scattering cross section for momentum transfer. 
$\delta$ is the average fractional energy lost per elastic collision $\frac{2 m}{M}$ where $m$ is the electron mass and $M$ is the mass of the gas particle.

$\gamma$ is $\frac{2 m}{3}\left(\frac{e E}{m}\right)^{2} e$ being the electronic charge and $E$ the rms electric field,

$$
\begin{gathered}
Q(u) \text { is } \sum_{i j} N_{i} u^{-3 / 2}(m / 2)^{-1 / 2} x \\
\int_{0}^{\infty}\left[\left(u^{\prime}+u_{i j}\right) \sigma_{i j}\left(u^{\prime}+u_{i j}\right) f\left(u^{\prime}+u_{i j}\right)-u^{\prime} \sigma_{i j}\left(u^{\prime}\right) f\left(u^{\prime}\right)\right] d u^{\prime}
\end{gathered}
$$

where $\sigma_{i j}$ and $N_{i}$ are as in (1) and $u_{i j}$ is the energy required to excite the state $u_{i j}$. The summation is overall inelastic states $j$ for each gas $i$.

$\alpha$ is a scaling parameter on the electric field expressed as

$$
\frac{\nu^{2}+\omega^{2}+\omega_{B}^{2}}{\left[\nu^{2}+\left(\omega-\omega_{B}\right)^{2}\right]\left[\nu^{2}+\left(\omega+\omega_{B}\right)^{2}\right]}
$$

where $\omega$ is the angular frequency of the exciting field $E$ and $\omega_{B}$ is the gyrofrequency of the electron in the earth's magnetic field $B$ where $\omega_{B}=\frac{e B}{m}$.

The solution of (3) is carried out numerically in order to obtain the desired distribution function, using experimental cross sections where available, and theoretically estimated ones elsewhere. A list of the cross sections used for these and other calculations has been given by Megill and Carleton [1964].

\section{Scaling Parameters}

While one may calculate the expected excitation for any given power density, excitation frequency, and collision frequency, the number of such calculations required to cover all possible cases is very large. We search, therefore, for scaling parameters in the theory which will allow more general application of calculated values.

If we investigate (3) in detail, we find that (after dividing through by $v(u)$ for convenience) the lefthand side of the equation constitutes a kind of "driving function" which describes the manner in which the electrons gain energy while the right-hand side describes the mechanisms which will cause the electrons to lose energy. We further see that on the right hand side of (3) only the parameters of the gas enter. The only reference to the mechanism which increases the electron energy then, is inside the brackets on the left hand side of the equation $(k T \delta+\gamma \alpha)$. Recall that we have divided the equation by $v(u)$.

The term $k T \delta$ presents a base energy (due to the neutral gas temperature) which is the electron energy for zero electric field. We have, then, as the driving function $\epsilon$ by which the electrons gain energy

$$
\epsilon=\frac{2}{3} m\left(\frac{e E}{m}\right)^{2} \frac{\nu^{2}+\omega^{2}+\omega_{B}^{2}}{\left[\nu^{2}+\left(\omega-\omega_{B}\right)^{2}\right]\left[\nu^{2}+\left(\omega+\omega_{B}\right)^{2}\right]} .
$$

Now let us examine expression (4) for limiting cases. In the ionosphere we have a rapidly decreasing number density with height so that the collision frequency $\nu=N \sigma v$ is also a rapidly varying function of height. Consider a beam of radiation extending upward through the ionosphere. It will, except for a small transition region, be in a situation in which $\nu>>\omega+\omega_{B}$ or $\nu<<\omega_{B}$. An exception is the condition $\omega=\omega_{B}$ about which more will be said later. We will, accordingly, consider these two cases in sequence.

When $\nu \ll \omega$ and if $\omega_{B}<\omega$,

$$
\epsilon=\frac{2}{3} \frac{e^{2}}{m}\left(\frac{E}{\omega}\right)^{2},
$$

which indicates that the mechanism by which the electrons gain energy is independent of the pressure of the gas and is only dependent upon its character as described by the right-hand side of (3).

On the other had, if $\nu \gg \omega \pm \omega_{B}$ then (4) reduces to

$$
\epsilon=\frac{2}{3} \frac{e^{2}}{m}\left(\frac{E}{\nu}\right)^{2}
$$

which, since $\nu \propto N$ and $N$ is proportional to the pressure, yields the scaling factor $E / P$ which is commonly used in gaseous electronics.

When $\omega=\omega_{B}$, gyroresonance exists. For purposes of calculating distribution functions, this may be considered to be the same as if there were a $\mathrm{d}-\mathrm{c}$ field. This is strictly true only if the radiation is circularly polarized in the extraordinary mode. For most purposes of interest in ionospheric problems, however, such an approximation is adequate.

In general, the electric field of importance in these calculations will be that present in the ionosphere at the height for which the calculations are being made. This field is, of course, dependent upon the power of the transmitter, the gain of the antenna and the absorption of the radiation below the point of interest. The calculation of the absorption of the radiation is in itself a difficult problem which is not discussed here.

We shall define two parameters then, in terms of which we shall present the data. These we will call the HF or high frequency and the LF or low frequency reduced power $\left(P_{r}\right)_{\mathrm{HF}}$ and $\left(P_{r}\right)_{\mathrm{LF}}$ where

$$
\left(P_{r}\right)_{\mathrm{HF}}=\frac{1}{\eta}\left(\frac{E}{\omega}\right)^{2}
$$

and

$$
\left(P_{r}\right)_{\mathrm{LF}}=\frac{1}{\eta}\left(\frac{E}{N}\right)^{2} .
$$

If $E$ is in volts per cm and $n$ is in ohms then these quantities have units of watts $\mathrm{cm}^{-2} \mathrm{sec}^{2}$ and watts 
$\mathrm{cm}^{-2} \mathrm{~cm}^{6}$ or watts $\mathrm{cm}^{4}$ if $N$ is in units of number per cubic centimeter. These units, while they may appear unwieldy, are convenient for application to geophysical problems.

The power required from an antenna in order to generate a specific effect may in turn be calculated from

$$
P_{\text {antenna }}=\frac{4 \pi\left(P_{r}\right)_{\mathrm{HF}}}{T G} h^{2} \cdot \omega^{2}
$$

or

$$
P_{\text {antenna }}=\frac{4 \pi\left(P_{r}\right)_{\mathrm{LF}}}{T G} h^{2} \cdot N^{2}
$$

where $h$ is the height at which the excitation rate is required, $N$ is the number density at that height and $G$ is the gain of the antenna. $T$ is the amount of power transmitted to height $h$. For the purposes of our further calculations $T$ is considered to be one.

\section{Observable Radiations}

Before calculating excitation rates of various states, let us first consider what radiations are likely to be visible from ground level. The first condition, of course, is that the experiment be done at night. Because we are dealing here with excitation by low energy electrons which already exist in the ionosphere as opposed to those created in a discharge we need consider radiation from states which can be excited from ground state atoms or molecules. The density of metastable states which can be excited to higher levels and will then radiate are negligible. Ground state rotational or vibrational states of the major molecular constituents will not radiate, and so, energy put into these states will not result in visible radiation. We discuss here only the excitation of electronic levels in the atoms and molecules which may possibly reradiate. In calculation of the distribution function, however, all inelastic states have been considered even though they are not discussed explicitly in this paper. In nitrogen, such states require so much energy to be excited (greater than $6.2 \mathrm{eV}$ ) that excitation is unlikely except in extremely powerful systems. In $\mathrm{O}_{2}$ the three most easily excited electronic states are the $a^{1} \Delta_{g}(0.98 \mathrm{eV})$, the $b^{1} \Sigma_{u}+(1.65 \mathrm{eV})$, and the $\mathrm{A}^{3} \Sigma_{u}{ }^{+}(4.47 \mathrm{eV})$. Of these, the first two have extremely small cross sections for direct excitation by electron collisions [Schulz and McDowell, 1962], ${ }^{1}$ so that we need to discuss only the excitation of the $\mathrm{A}^{3} \Sigma_{u}{ }^{+}$state which radiates in the Herzberg bands. These bands are seen in the night airglow and are rendered difficult to observe because the energy is distributed into many lines in the band. However, fairly small amounts of excitation should be detectable with sophisticated techniques.

1 These states are, of course, excited to some extent; but with the method of excitation considered here, we find it will never, at any power less than that which is required for breakdown, be possible to excite a detectable amount of radiation from them.
This leaves the ${ }^{1} \mathrm{D}(1.96 \mathrm{eV})$ and ${ }^{1} \mathrm{~S}(4.17 \mathrm{eV})$ states of atomic oxygen. The former would be undetectable because it is strongly deactivated by collisions with $\mathrm{O}_{2}$ at altitudes below about $140 \mathrm{~km}$. These calculations do not apply in any practical situation for altitudes above $140 \mathrm{~km}$ because the dominant energy loss term there would be that due to diffusion out of the exciting beam, an effect which is not included in the calculation of the distribution function. $\mathrm{O}\left({ }^{1} \mathrm{~S}\right)$ is the strongest line of the normal night airglow and variations of intensity should be readily observed if excitation is accomplished. This leaves then, as the two most readily observed emissions $\mathrm{O}_{2}\left(\mathrm{~A}^{3} \Sigma_{u}{ }^{+}\right)$and $\mathrm{O}\left({ }^{1} \mathrm{~S}\right)$ both of which require more than $4 \mathrm{eV}$ for excitation. The generation of electrons of this energy is particularly difficult in air because there is a very large cross section for energy loss to the vibrational states of $\mathrm{N}_{2}$ for all electrons between about 1.7 and $4 \mathrm{eV}$ [Schulz, 1959]. It is of interest to know, however, just what power would be required in order to obtain detectable intensities. In figures $1 \mathrm{a}$ and $1 \mathrm{~b}$, we plot specific rates of excitation for the above two states, as well as two body attachment rates and ionization rates, as a function of the two previously defined reduced powers. In order to obtain the excitation rate at a particular power and height, one needs to multiply the value given in the figure by electron density and constituent density. The cross section for the excitation of the $\mathrm{O}_{2}\left(\mathrm{~A}^{3} \Sigma_{u}{ }^{+}\right)$is estimated from the data of Schulz [1962], that for the excitation of $\mathrm{O}\left({ }^{1} \mathrm{~S}\right)$ from the theoretical calculations of Seaton [1953]. As an example let us suppose that we wish to know the power required to excite 100 rayleighs $^{2}$ of $5577 \mathrm{~A}$ radiation (from $\mathrm{O}\left({ }^{1} \mathrm{~S}\right)$ in a slab of the ionosphere between 90 and $100 \mathrm{~km}$ ). Then this means that we must excite 100 atoms $/ \mathrm{cm}^{3} / \mathrm{sec}$, which will later radiate. If we assume that there are on the average 500 electrons $/ \mathrm{cm}^{3}$ and $2 \times 10^{12}$ oxygen atoms $/ \mathrm{cm}^{3}$ in the slab, then we would expect to see approximately 100 rayleighs for a specific excitation rate of $\mathrm{O}\left({ }^{1} \mathrm{~S}\right)$ equal to $10^{-13}$ which requires from figure 1 a a reduced HF power of $3.3 \times 10^{-21} \mathrm{~W}$ sec. This results (using $5 \mathrm{a})$ in a required power flux of $8 \times 10^{-5} \mathrm{~W} / \mathrm{cm}^{2}$, if we are considering a transmitter at $50 \mathrm{Mc} / \mathrm{s}$. Using (6a) it is found that $9 \times 10^{10} \mathrm{~W}$ are required for an isotropic radiator. If we have an antenna gain of $10^{4}$, we then need $9 \mathrm{MW}$ transmitted from the antenna. The very large power required for minimum detection conditions indicates that experiments resulting in the excitation of optical radiation may not be economically feasible unless the installation can be justified for other applications.

An additional consideration should be made at this point. The calculations made here yield average excitation rates while the power is turned on, and after a steady state has been established. The time required to establish equilibrium is approximately equal to the product of the average fractional energy loss per collision (averaged over all types of collisions)

${ }^{2}$ The rayleigh is defined as $10^{3}$ emissions per $\mathrm{cm}^{2}$ column per second through the radiating medium. 


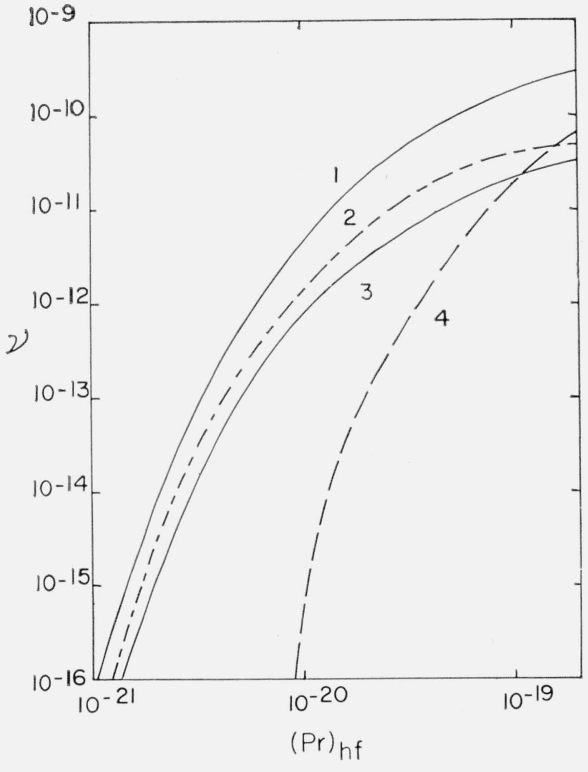

FIGURE 1a. This figure shows (1) the rate of excitation of $\mathrm{O}\left({ }^{1} \mathrm{D}\right)$, (2) the rate of attachment to $\mathrm{O}_{2},(3)$ the rate of excitation of $\mathrm{O}_{2}\left(\mathrm{~A}^{3} \Sigma_{\mathrm{u}}^{+}\right)$, and (4) the rate of ionization as a function of reduced power with high frequency excitation.

To obtain an absolute rate the ordinate should be multiplied by the constituent density and the electron density. The power flux in watts $\mathrm{cm}^{-2}$ may be had by multiplying the abscissa by $(2 \pi f)^{2}$ where $f$ is the frequency of excitation. These curves should be used if $\nu<\omega \pm \omega_{b}$.

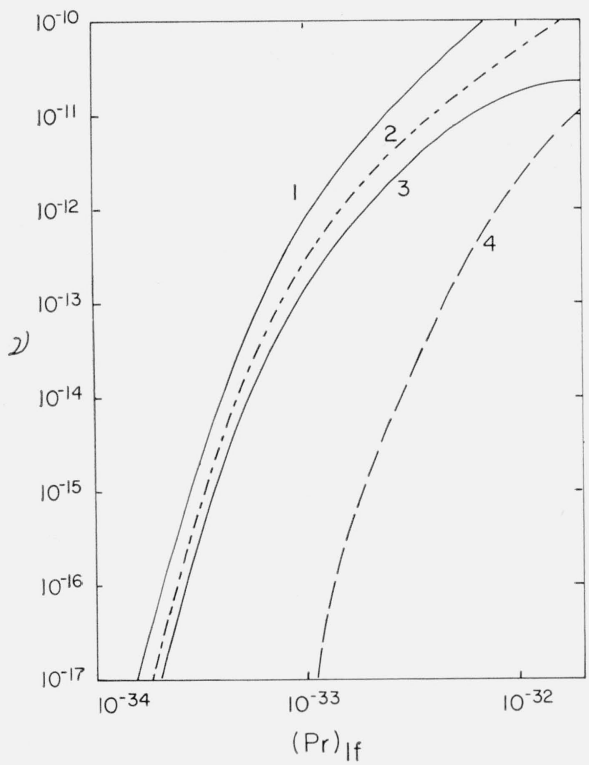

Figure 1b. This figure shows the same quantities as figure 1 a except that they are plotted versus the reduced power for low frequency excitation.

In this case the abscissa should be multiplied by the square of the total number density in particles per $\mathrm{cm}^{3}$ to yield watts $\mathrm{cm}^{-2}$. These curves should be used when $\nu>\omega \pm \omega_{b}$ and for the gyroresonance condition. and the collision frequency. For air the average energy loss is not constant with energy, but for rough calculations may be assumed to be of the order of $10^{-3}$. For the convenience of the reader and those who might wish to extend the calculations to include absorption we plot in figures $2 \mathrm{a}$ and $2 \mathrm{~b}$ the average collision frequency, normalized to one particle $/ \mathrm{cm}^{-3}$ and the average energy against the two previously defined reduced powers. The relaxation time will vary from the order of a microsecond at 60 $\mathrm{km}$ height to the order of a millisecond at $100 \mathrm{~km}$ height. Transmitters must, therefore, be operated with pulse lengths long compared to these times for steady state to be achieved. For the higher heights this puts severe requirements on the transmitter because of the long pulses required.

One other parameter which must be considered for the case of $0\left({ }^{1} \mathrm{~S}\right)$ is that the state has a mean life of the order of 1 sec. For this reason the effect of

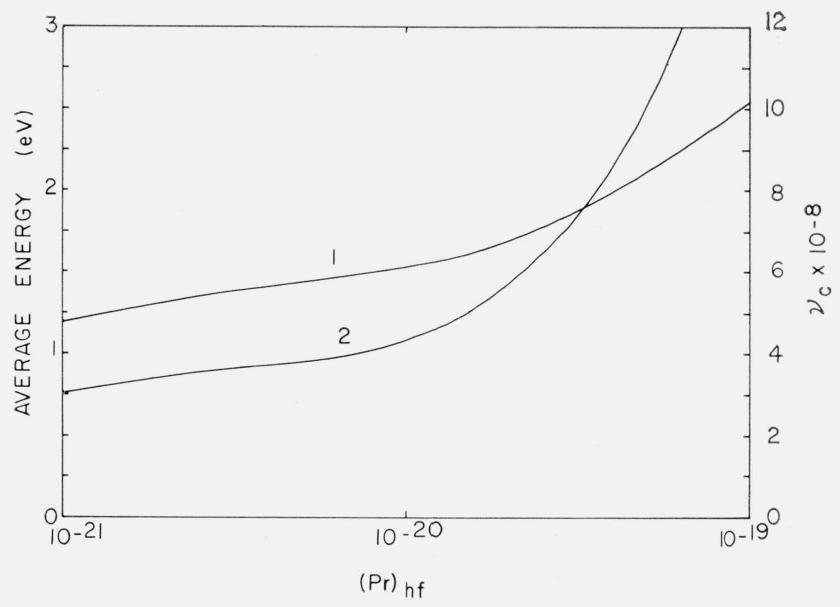

Figure 2a. The abscissa is as defined in figure 1a while the ordinates are the average collision frequency (curve 2) per neutral particle in $\sec ^{-1}$ and the average energy in $\mathrm{V} V$ (curve 1).

The averages are over the calculated distribution functions.

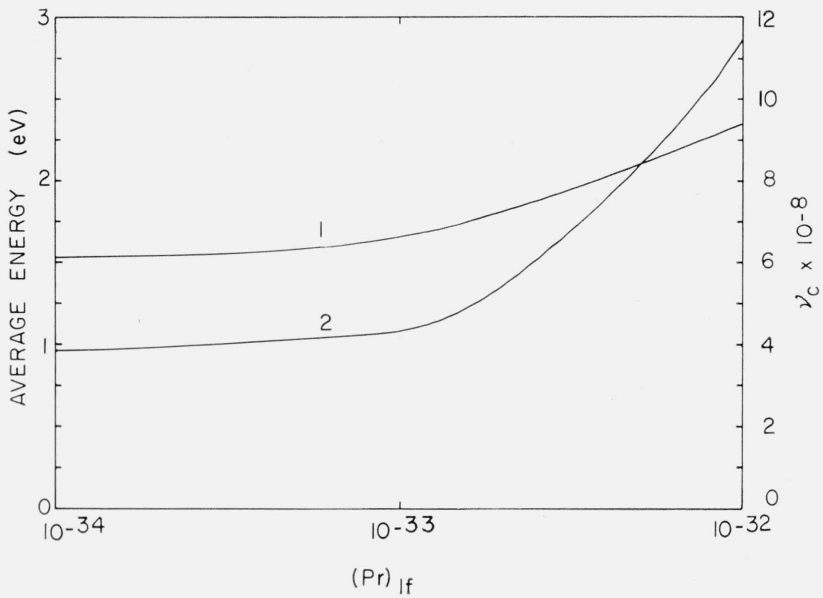

FIgURE 2b. The abscissa is as defined in figure $1 b$ while the ordinates are as defined in figure $2 a$. 
pulsed operation will be to reduce the observed change in intensity by the duty cycle. It is to be noted that this does not mean that the average power flux is the important quantity. The extremely nonlinear nature of the intensity versus power curve indicates that the transmitter should be operated at as high a peak power as possible and then for as high a duty cycle as is compatible with this condition.

\section{Modification of the Ionosphere by Attachment}

Another possible experiment of interest is the modification of the atmosphere by attaching the electrons to $\mathrm{O}_{2}$ molecules. The problem has been discussed in some detail by Molmud [1964] for attachment by three body attachment,

$$
\mathrm{O}_{2}+e+M \rightarrow \mathrm{O}_{2}{ }^{-}+M .
$$

The rate of this process is probably the easiest one to change with transmitted RF power because there is a maximum in the rate at about $0.1 \mathrm{eV}$ average electron energy [Chanin, Phelps, and Biondi, 1959].

There is a second process which occurs at higher energies. This is dissociative attachment of $\mathrm{O}_{2}$, by the following reaction,

$$
\mathrm{O}_{2}+e \rightarrow \mathrm{O}^{-}+0 \text {. }
$$

This process has a threshold at $4 \mathrm{eV}$ with a maximum at $6.3 \mathrm{eV}$ [Schulz, 1962]. We may calculate this rate in the same manner that one calculates attachment rates. These rates are given in figures 1a and 1b. In the example previously discussed, if we were exciting 100 rayleighs of the 5577 A line we would expect electrons to attach at a rate of 3 percent per second. The electrons would effectively disappear at this rate until they are re-released either by collisional detachment or by the reverse of process (8). These rates are not well known and it might be that an ionospheric experiment would be one of the best with which to determine their rates.

\section{Breakdown in the Ionosphere}

The general technique of calculation used here can, in principle, be used to compute the power required to "breakdown" the ionosphere. In the lower ionosphere one may, to a large extent, ignore diffusion losses and may therefore compute the rate of attachment as a function of power and the rate of ionization as a function of power. As the ionization rate becomes the larger the ionization density will grow exponentially until some limiting process sets in. The details of an approximate treatment of some of these processes is given by Lombardini [1964]. In addition he gives comparisons to experimental data which indicate the order of the accuracy of the calculations. Specific rates of ionization as a function of $\left(P_{r}\right)_{H F}$ and $\left(P_{r}\right)_{L F}$ are shown in figures $1 \mathrm{a}$ and $1 \mathrm{~b}$.

\section{7. $F$-Region Emissions}

In the investigation of possible modification of airglow intensities another possibility of interest presents itself in terms of the $F$-region emission from $\mathrm{O}\left({ }^{1} \mathrm{D}\right)$ at $6300 \AA$. This radiation is presumably due to the two step recombination of $\mathrm{O}^{+}$by means of

$$
\mathrm{O}^{+}+\mathrm{N}_{2} \rightarrow \mathrm{NO}^{+}+\mathrm{N}
$$

and

$$
\mathrm{NO}^{+}+e \rightarrow \mathrm{N}+\mathrm{O}\left({ }^{1} \mathrm{D}\right) .
$$

The rate of (9) is the slower and controls the emission rate so that the intensity of $6300 \AA$ radiation is proportional to the product of the $\mathrm{O}^{+}$and $\mathrm{N}_{2}$ densities.

Farley [1963] has shown that by using power at the plasma frequency it is possible with reasonable power to heat $(\sim 250 \mathrm{~kW})$ the electrons enough that the enhanced diffusion decreases the electron and ion density by about 10 percent or more. This should, in turn, decrease the intensity of $6300 \AA \mathrm{ra}-$ diation by 10 percent and serve as an experimental check on the recombination process in the $F$ region. It should be noted that the mean life of the $\mathrm{O}\left({ }^{1} \mathrm{D}\right)$ state is $100 \mathrm{sec}$, so that a transmitter which will perform the described experiment must be operated CW.

\section{Conclusions}

The requirements on large power transmitters which can be expected to yield observable amounts of optical radiation have been expressed quantitatively for the two most likely radiations. We find that the size of the required installations is so large that such experiments may not be economically feasible at the present time.

The author recognizes the very considerable contributions of Mrs. J. D. Droppleman in the performance of the calculations and the preparation of the figures.

\section{References}

Bailey, V. A. (1959), Some possible effects caused by strong gyrowaves in the ionosphere. J. Atmospheric Terrestial Phys. 14, 229-324.

Carleton, N. P., and Lawrence R. Megill (1962), Electron energy distribution in slightly ionized air under the influence of electric and magnetic fields, Phys. Rev. 126, 2089-2099.

Chanin, L. M., A. V. Phelps, and M. Biondi (1959), Measurements of the attachment of slow electrons in oxygen, Phys. Rev. Let. 2, 344.

Clavier, P. A. (1961), Man-made heating and ionization in the upper atmosphere, J. Appl. Phys. 32, 570-577 and erratum 2651.

Farley, D. T. (1963), Artificial heating of electrons in the $F$ region of the ionosphere, J. Geophys. Res. 68, 401-413. 
Lombardini, P. P. (1964), Alteration of the electron density of the lower ionosphere with ground based transmitters. presented at the Nonlinear Conference, Dec. 16-17, 1963, National Bureau of Standards. Poulder, Colo. NES Tech. Note $211, \mathbf{5}, 87-110$.

Megill, Lawrence R., and J. H. Cahn (1964), Calculation of electron energy distribution functions in the ionosphere, to be published.

Megill, Lawrence R., and N. P. Carleton (1964), Excitation by local electric fields in the aurora and airglow, J. Geophys. Res. 69, 101-122.

Molmud, P. (1964), Use of radio transmitters to decrease $D$ region electron density, presented at the Nonlinear Conference, Dec. 16-17, 1963, National Bureau of Standards, Boulder, Colo. NBS Tech. Note $211,6,1-88$.
Schulz, G. J. (1959), Measurement of excitation of $\mathrm{N}_{2}$, CO, and $H_{e}$ by electron impact, Phys. Rev. 116, 1141-1147.

Schulz, G. J. (1962), $\mathrm{O}_{2}, \mathrm{CO}$, and $\mathrm{CO}_{2}$ by electron impact, Phys. Rev. 128, No. 1, 178-186.

Schulz, G. J., and J. T. Mc Dowell (1962), Excitation of vibrational and electronic levels in $\mathrm{O}_{2}$ by electronic impact, Phys. Rev. 128, No. 1, 174-177.

Seaton, M. J. (1953), The Hartree-Fock equations for continuous states with application to electron excitation of ground configuration terms of OI, Phil. Trans. Roy. Soc. 245, 469 .

(Paper 69D1-444) 